\title{
Atemmuskeltraining: State-of-the-Art
}

\author{
Respiratory Muscle Training: State of the Art
}

Autoren

Institute
O. Göhl' , D. J. Walker ${ }^{2}$, S. Walterspacher ${ }^{2}$, D. Langer ${ }^{3}$, C. M. Spengler ${ }^{4}$, T. Wanke ${ }^{5}$, M. Petrovic ${ }^{5}$, R.-H. Zwick ${ }^{6}$, S. Stieglitz ${ }^{7}$, R. Glöckl ${ }^{8}$, D. Dellweg ${ }^{9}$, H.-J. Kabitz ${ }^{2}$

Die Institutsangaben sind am Ende des Beitrags gelistet. eingereicht 18.8.2015 akzeptiert nach Revision 10.11.2015

\section{Bibliografie}

Dol http://dx.doi.org/ 10.1055/s-0041-109312 Pneumologie 2016; 70: 37-48 (c) Georg Thieme Verlag KG Stuttgart · New York ISSN 0934-8387

\section{Korrespondenzadresse} Dr. phil. Oliver Göhl Rehaklinik HeidelbergKönigstuhl Kohlhof 8

69117 Heidelberg o.goehl@rehaklinikkoenigstuhl.de

\section{Zusammenfassung \\ $\nabla$}

Spezifisches Atemmuskeltraining (IMT) ist in der Lage, die Funktion der Atemmuskulatur zu verbessern. Anhand der Studienlage und klinischen Praxis haben sich 3 Methoden etabliert: 1.) kontrollierte Stenoseatmung („resisitive load“), 2.) „threshold load“ und 3.) normokapnische Hyperpnoe. Die jeweiligen Methoden und zugehörigen Trainingsgeräte weisen spezifische Charakteristika auf. Die Einleitung eines IMT erfordert eine vorausgehende Diagnostik der Atemmuskelfunktion und eine detaillierte individualisierte Trainingseinweisung und -anleitung.

Ziel dieser Übersichtsarbeit ist es, anhand der aktuell verfügbaren Literatur die unterschiedlichen IMT-Methoden bei den wichtigsten Indikationsgebieten zu beleuchten und kritisch zu diskutieren. Bei den neuromuskulären Erkrankungen werden Muskeldystrophien, spinale Muskelatrophien, amyotrophe Lateralsklerose, Phrenicusparese und Verletzungen des Rückenmarks dargestellt. Zudem werden die Bereiche interstitielle Lungenerkrankungen, Sarkoidose, Linksherzinsuffizienz, pulmonal-arterielle Hypertonie, Kyphoskoliose und Adipositas beleuchtet. Bei den obstruktiven Atemwegs- und Lungenerkrankungen werden COPD, Asthma, Mukoviszidose und „Non-CFBronchiektasen“ aufgearbeitet. Abschließend finden sich Ausarbeitungen für den Bereich Weaning vom Respirator und im Zusammenhang mit körperlicher Aktivität.

\section{Hintergrund}

$\nabla$

Die Atempumpe bildet das Kernstück des ventilatorischen Systems, welches den An- und Abtransport der Atemgase sicherstellt ( Abb.1) [1,2]. Sie ist durch ein empfindliches Gleichgewicht zwischen der verfügbaren Kapazität und der ihr auferlegten Last gekennzeichnet ( $\bullet$ Abb.2) $[1,2]$. Einschränkungen der Zwerchfellfunktion oder

\section{Abstract \\ $\nabla$}

Specific respiratory muscle training (IMT) improves the function of the inspiratory muscles. According to literature and clinical experience, there are 3 established methods: 1.) resistive load 2.) threshold load and 3.) normocapnic hyperpnea. Each training method and the associated devices have specific characteristics. Setting up an IMT should start with specific diagnostics of respiratory muscle function and be followed by detailed individual introduction to training.

The aim of this review is to take a closer look at the different training methods for the most relevant indications and to discuss these results in the context of current literature.

The group of neuromuscular diseases includes muscular dystrophy, spinal muscular atrophy, amyotrophic lateral sclerosis, paralysis of the phrenic nerve, and injuries to the spinal cord. Furthermore, interstitial lung diseases, sarcoidosis, left ventricular heart failure, pulmonary arterial hypertension (PAH), kyphoscoliosis and obesity are also discussed in this context. COPD, asthma, cystic fibrosis (CF) and non-CF-bronchiectasis are among the group of obstructive lung diseases. Last but not least, we summarize current knowledge on weaning from respirator in the context of physical activity. 


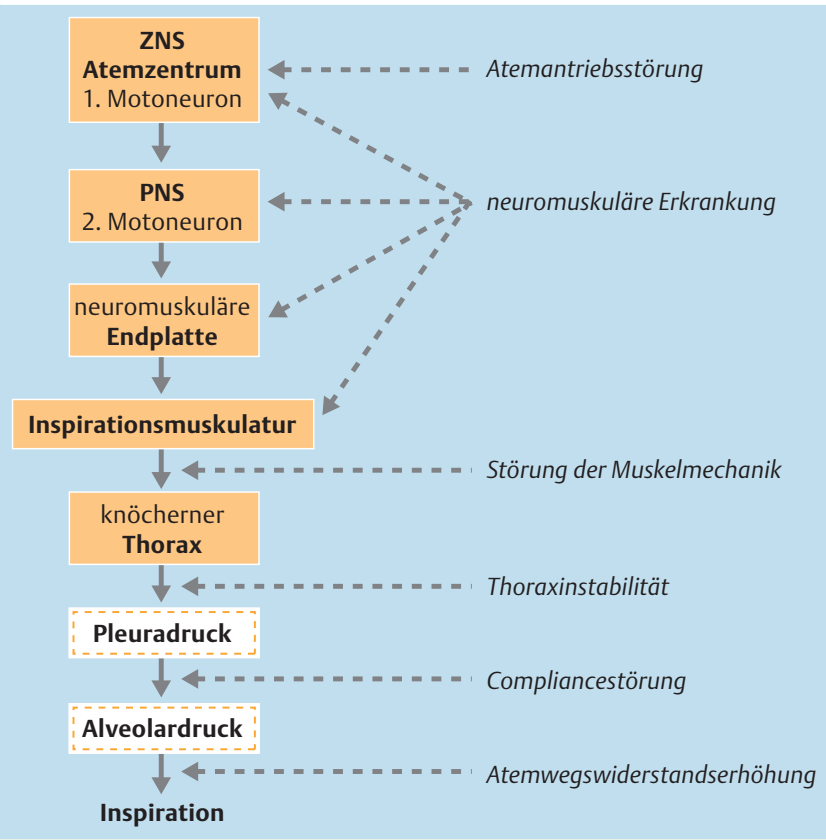

Abb.1 Die Atempumpe mit unterschiedlichen Lokalisationen möglicher Funktionsstörungen [2].

der Atemmuskelfunktion ist zu klären, ob eine Einschränkung der Atemmuskulatur und/oder erhöhte Beanspruchung vorliegen und wie ausgeprägt diese ausfallen [1]. In dieser Übersichtsarbeit wird die Beeinflussung der Funktion der (inspiratorischen) Atemmuskulatur durch ein systematisches Training bei unterschiedlichen Voraussetzungen und Krankheitsbildern dargestellt.

\section{Atemmuskeltraining}

Die Atemmuskulatur besteht aus quergestreifter Muskulatur. Sie unterliegt daher denselben physiologischen Gesetzmäßigkeiten wie die übrige Skelettmuskulatur [3] und ist grundsätzlich bzgl. Kraft und Ausdauer trainierbar [4-6]. Für beide Modalitäten ist eine exakte Dosierung der Trainingslast („targeting“) erforderlich. Aufgrund (patho-) physiologischer Überlegungen kommt dem inspiratorischen Atemmuskeltraining (IMT) gegenüber dem exspiratorischen Atemmuskeltraining (EMT) bei Weitem die gröBere Bedeutung zu.

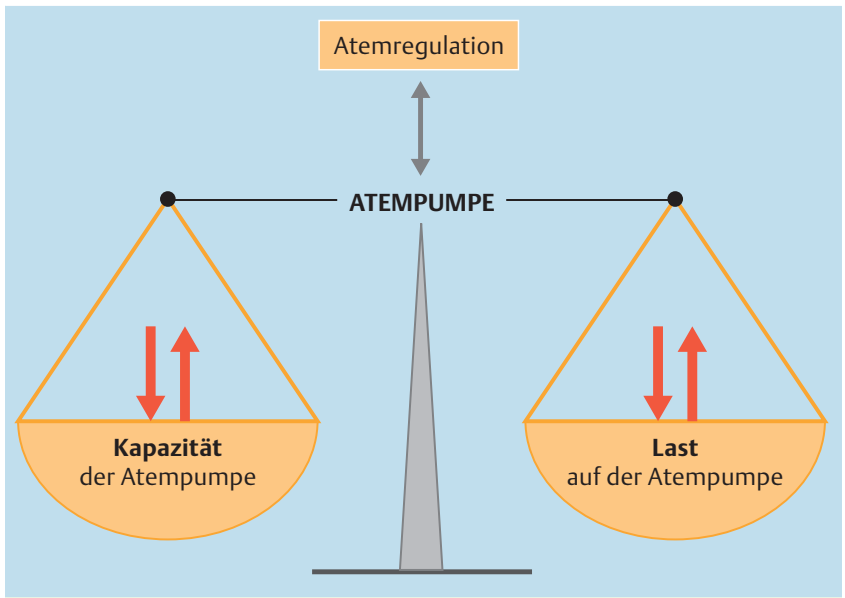

Abb.2 Die Atempumpe und das empfindliche Gleichgewicht zwischen der ihr auferlegten Last und ihrer Kapazität [2].

\section{Methoden}

$\checkmark$

Anhand der verfügbaren Literatur und der klinischen Erfahrung haben sich die drei nachfolgend aufgeführten Trainingsmethoden etablieren können [5-8] (vgl. @ Tab.1):

- kontrollierte Stenoseatmung („resistive load“) und „threshold load“ als Methoden des primär auf Kraftsteigerung abzielenden IMT: Kontraktionen (inspiratorisch) mit hohem Kraftaufwand und wenigen Wiederholungen.

- „Normokapnische Hyperpnoe“ als „Ausdauertraining“: Kontraktionen (in- und exspiratorisch) mit niedrigem Kraftaufwand und vielen Wiederholungen.

\section{Resistive Load}

Zur Erzeugung eines spezifischen trainingswirksamen Widerstands, der mindestens 30\% der maximal möglichen Inspirationskraft („PImax“) betragen sollte [5], wird durch eine definierte Stenose geatmet. Da hier der Widerstand vom aufgebauten Fluss abhängig ist, sollten die Trainingsgeräte den Atemfluss kontrollieren und einen Feedbackmechanismus besitzen („targeted resistive breathing“), der eine korrekte Durchführung des Trainings anzeigt [9]. Die neueste Generation dieser Geräte besitzt adaptive Stenosen, welche sich elektronisch dem Atemfluss anpassen. Hier wird jedoch zudem ein definierter Anfangswiderstand eingebaut, weshalb diese Geräte auch Komponenten des „threshold load“ (siehe unten) aufweisen.

Tab.1 Charakteristika der verschiedenen Trainingsmethoden und Devices.

\begin{tabular}{|c|c|c|c|c|c|}
\hline Trainingsmethode & Fokus & Kosten & Feedbacksystem & $\begin{array}{l}\text { Speicherung } \\
\text { Trainingsdaten }\end{array}$ & Hersteller \\
\hline threshold Load & Kraft und Ausdauer & gering & nicht erforderlich & nein & $\begin{array}{l}\text { z. B. Threshold IMT }{ }^{\circledR} \text {; } \\
\text { POWERbreathe }{ }^{\circledR} \text { Medic }\end{array}$ \\
\hline $\begin{array}{l}\text { resistive load: } \\
\text { kontrollierte Stenoseatmung }\end{array}$ & Kraft und Ausdauer & teuer & optisch & ja & z. B. Respifit $S^{\circledR}$ \\
\hline $\begin{array}{l}\text { resistive load: } \\
\text { adaptive Stenoseatmung }\end{array}$ & Kraft und Ausdauer & moderat & optisch & ja & z. B. Powerbreathe (K-Serie) \\
\hline normokapnische Hyperpnoe & Ausdauer & teuer & optisch/akkustisch & ja & z. B. Spirotiger ${ }^{\circledR}$ \\
\hline
\end{tabular}




\section{Threshold Load}

Bei der Methode "threshold load“ muss zunächst statisch ein inspiratorischer Widerstand aufgebaut werden, bis eine vorher definierte Schwelle (engl.: threshold) erreicht wird. Anschließend ist eine Inspiration gegen den gleichbleibenden Widerstand möglich $[10,11]$. Der so erzeugte Widerstand, der mindestens $30 \%$ des PImax entsprechen sollte [5], ist unabhängig vom Atemfluss des Patienten. Daher ist zur korrekten Durchführung des Trainings kein Feedback-System erforderlich.

\section{Normokapnische Hyperpnoe}

Hier erfolgt eine schnelle und tiefe Ein- und Ausatmung. Um eine Hyperventilation mit konsekutiver Hypokapnie zu verhindern, wurden Trainingsgeräte entwickelt, welche durch partielle Rückatmung der Atemluft eine Normokapnie gewährleisten. Die Durchführung des Trainings ist koordinativ anspruchsvoll, sodass eine intensive Trainingseinweisung der Patienten notwendig ist; ein optisches/akustisches Feedback-System ist obligat. Anzustreben sind Trainingsintensitäten, die etwa $60 \%$ des Atemgrenzwertes (z.B. maximal voluntary ventilation; MVV) eines Patienten entsprechen $[5,12,13]$.

\section{IMT-Geräte}

Bisher existieren zu wenig publizierte Daten, um den Einsatz der verschiedenen IMT-Methoden bei Patienten direkt miteinander zu vergleichen. Es können somit aktuell nur mit Einschränkung Empfehlungen gegeben werden, bei welchem Patientenkollektiv welche Methode eingesetzt werden sollte.

\section{Umsetzung}

$\nabla$

Die Initiierung bzw. Optimierung eines IMT kann z.B. ideal im Rahmen eines pneumologischen Rehabilitationsprogrammes umgesetzt werden ( $\odot$ Tab.2). Zu Beginn sollten die Patienten über die physiologischen Veränderungen der Atemmuskulatur und die daraus resultierenden Prinzipien des IMT aufgeklärt werden. Des Weiteren sollten die Wirkmechanismen sowie die zu erwartenden Effekte vermittelt werden. Bei der Einweisung in das jeweilig verwendete Trainingsgerät sollten die Patienten neben Handhabung und Bedienung des Gerätes auch Informationen über die notwendigen Hygiene- und Reinigungsmaßnahmen erhalten. Weiterführende Informationen sind z. B. auf der Homepage www.atemmuskeltraining.com verfügbar.

\section{IMT bei restriktiven Ventilationsstörungen}

\section{Neuromuskuläre Erkrankungen}

Hierunter versteht man eine heterogene Gruppe von Krankheiten, welche nach der Ätiologie, dem klinischen Erscheinungsbild sowie der Lokalisation der Störung klassifiziert werden (vgl. - Tab.3, $\triangle$ Abb.3, $\odot$ Tab.4).

Wesentliches gemeinsames Kriterium ist die zumeist progrediente Muskelschwäche unterschiedlicher Geschwindigkeit und Ausprägung, die mit zunehmendem Lebensalter u.a. zu einer Atemmuskelschwäche und konsekutiv zur hyperkapnischen respiratorischen Insuffizienz führt.
Tab.2 Trainingsparameter eines initialen IMT Aufbauprogrammes.

\begin{tabular}{|ll|}
\hline Häufigkeit & 5-7-mal pro Woche \\
\hline Dauer & 7-mal 2 Minuten (mit je 1 Minute Pause) \\
\hline Intensität & $\begin{array}{l}\text { initial ca. 30\% PImax (kontinuierliche } \\
\text { Progression wie individuell möglich) }\end{array}$ \\
\hline $\begin{array}{l}\text { Trainingssteuerung } \\
\text { (mod. Borg-Skala 0-10) }\end{array}$ & Atemkraftanstrengung: 4-6 \\
\hline
\end{tabular}

PImax=maximaler inspiratorischer Mundverschlussdruck

Tab.3 Übersicht der neuromuskulären Erkrankungen (mod. nach [15]).

\begin{tabular}{|c|c|}
\hline \multicolumn{2}{|c|}{ Erkrankungen des Muskels (Myopathien) } \\
\hline Muskeldystrophie & $\begin{array}{l}\text { z. B. Typ Duchenne, Typ Becker, Glieder- } \\
\text { gürteldystrophie }\end{array}$ \\
\hline myotone Muskelerkrankung & z. B. myotone Dystrophie \\
\hline entzündliche Myopathien & z. B. Polymyositis, Dermatomyositis \\
\hline metabolische Myopathien & z. B. M. Pompe \\
\hline $\begin{array}{l}\text { Erkrankung der neuro- } \\
\text { muskulären Endplatte }\end{array}$ & z. B. Myasthenia gravis \\
\hline $\begin{array}{l}\text { Erkrankung des zentralen/ } \\
\text { peripheren Nervensystems }\end{array}$ & $\begin{array}{l}\text { z. B. ALS (Amyotrophe Lateralsklerose), } \\
\text { spinale Muskelatrophie, Phrenicusparese }\end{array}$ \\
\hline
\end{tabular}

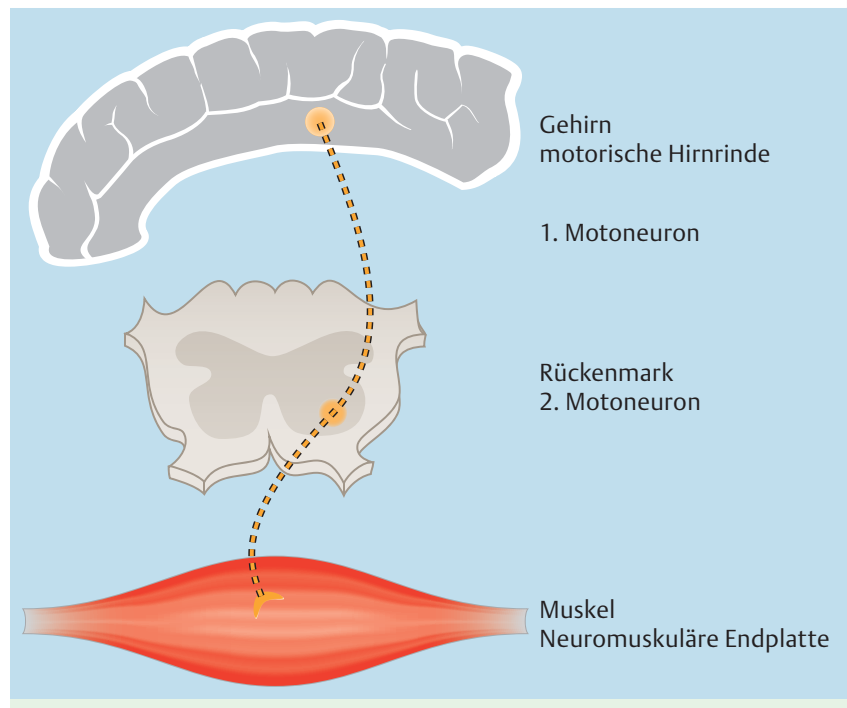

Abb.3 Das motorische Nervensystem [14].

\section{Muskeldystrophien}

Die zwei häufigsten Muskeldystrophien (beide „BeckengürtelTyp“) sind: Typ Duchenne („maligne“) und Typ Becker-Kiener („benigne“) [16]. IMT in Form von resistive load erzielte hier Verbesserungen der Kraft als auch der Ausdauer der Atemmuskulatur sowie der Lebensqualität $[17,18]$. Es besteht eine Dosis/Wirkungs-Beziehung $[19,20]$. IMT erfolgte hierbei favorisiert täglich mit 70\% des PImax; allerdings nur bei Patienten mit einer Vitalkapazität $>11$ (bzw. 25\%soll) [17,18]. Das IMT sollte so früh wie möglich begonnen werden und zeigt auch im Langzeitverlauf günstige Ergebnisse [21].

Anhand der dünnen Datenlage zeigten sich bei diesem Patientenkollektiv weder für "normokapnische Hyperpnoe“ noch „threshold load" signifikante Verbesserungen in Bezug auf die Atemmuskelkraft sowie die Vitalkapazität [22,23]. 


\section{Spinale Muskelatrophien}

Dies sind Erkrankungen, die auf einem Untergang der Vorderhornzellen im Rückenmark (zweites motorisches Neuron) beruhen. Publiziert sind lediglich Studien mit Verwendung von „resistive load“ $[18,21,24]$. Hierbei verbesserte sich sowohl die Atemmuskelkraft als auch die Dyspnoe $[18,21]$. Ein anhaltender Trainingseffekt konnte bei Kindern (bis zum 14. Lebensjahr) für 3 (Atemmuskelkraft) bzw. 12 Monate (Dyspnoe) erreicht werden [24].

\section{Amyotrophe Lateralsklerose (ALS)}

Bei dieser zumeist rasch progressiven Erkrankung degenerieren das 1. und 2. Motorneuron: Es kommt u.a. zu progredienten muskulären Funktionseinbußen - auch der Atemmuskulatur. Aktuell existieren nur wenige kontrollierte Studien zum IMT bei ALS. Treshold load verbessert hier die MVV [25], die Atemmuskelkraft kann (kurzfristig) minimal gesteigert werden [26]. Aussagen zur Beeinflussung der Lebensqualität und des weiteren Krankheitsverlaufs sind aktuell nicht möglich. Aufgrund der raschen Progredienz der Erkrankung erscheint der Stellenwert des IMT hierbei jedoch sehr fraglich - ggf. muss auch mit einer klinischen Verschlechterung gerechnet werden.

\section{Phrenicusparese}

Tägliches IMT mittels resistive load (70\% PImax) zeigte hier positive Effekte mit einer möglicherweise günstigen Beeinflussung auch beim Vorliegen einer respiratorischen Insuffizienz [27].

\section{Verletzungen des Rückenmarks (hoher Querschnitt)}

Zwar rechnen diese Erkrankungen streng genommen nicht zu den neuro-muskulären, zeigen jedoch ähnliche klinische Auswirkungen. Die meisten Studien verwendeten hier normokapnische Hyperpnoe [28, 29]. Trainingsdauern reichten von 10-20 Minuten, wobei $40 \%$ des MVV bei Tetraplegikern und $60 \%$ des MVV bei Paraplegikern gewählt wurden [28]. Eine Metaanalyse zeigte, dass sowohl die Vitalkapazität als auch die Atemmuskelkraft verbessert werden konnten [30].

\section{Sonstige restriktive Ventilationsstörungen}

Interstitielle Lungenerkrankungen (ILD)

Hierbei wird die Ausbildung einer hyperkapnischen respiratorischen Insuffizienz zumeist erst in sehr späten Stadien beobachtet [31]. Gleichwohl kann IMT hierbei den PImax und die 6-Minuten-Gehstrecke verbessern sowie die Dyspnoe reduzieren [32] (vgl. $\odot$ Tab.5).

\section{Sarkoidose}

Luftnot gehört neben Erschöpfung („Fatigue“) zu den häufigsten Symptomen bei Sarkoidose [33]. Eine Beteiligung der Atemmuskulatur mit Funktionseinschränkung ist möglich [34]. Studien zum gezielten Einsatz eines IMT bei Sarkoidose existieren aktuell nicht.

\section{Kyphoskoliose}

Die Atemmuskelstärke ist bei milden Formen zumeist normal, fällt bei schweren Verlaufsformen jedoch ab [35]. Es existieren lediglich einzelne Fallberichte zum Einsatz des IMT [36].

\section{Adipositas}

Die Atemmuskelkraft ist hier zumeist normal oder aber gering reduziert $[37,38]$. IMT mittels normokapnischer Hyperpnoe verbesserte die Vitalkapazität, die Ausdauer der Atemmuskulatur, die 6-Minuten-Gehstrecke sowie die Dyspnoe und Lebensqualität; nicht jedoch die Atemmuskelstärke [39]. Mittels „threshold load“ konnten der PImax sowie das MVV gesteigert werden [40]. Im Vorfeld einer bariatrischen Chirurgie kann IMT den PImax erhöhen und eine postoperative Abnahme verringern [41]. Postoperatives IMT verbessert deutlich die Atemmuskelkraft sowie die Ausdauer [42].

\section{IMT bei kardio-zirkulatorischen Störungen}

\section{Linksherzinsuffizienz}

Die Atemmuskelkraft ist hierbei reduziert, stellt ein unabhängiges Mortalitätsrisiko dar und korreliert mit Dyspnoe, maximaler Sauerstoffaufnahme und funktionellem Stadium („NYHAKlasse“) $[43,44]$. IMT verbessert den PImax, die körperliche Belastbarkeit und reduziert die Dyspnoe [45-48] (vgl. @ Tab.6).

\section{Pulmonal-arterielle Hypertonie (PAH)}

Hier mehren sich die Hinweise, dass die Atemmuskelfunktion eingeschränkt ist [49]. Rehabilitative Maßnahmen, die auch ein respiratorisches Training beinhalten, führen zu einer Zunahme der 6-Minuten-Gehstrecke wie auch der maximalen Sauerstoffaufnahme [50,51]. Isoliertes IMT bei PAH wurde bisher nicht untersucht (vgl. @ Tab.6).

\section{Obstruktive Atemwegs- und Lungenerkrankungen $\nabla$ COPD}

Hier besteht bzgl. des IMT eine sehr gute Datenlage (vgl. @ Tab. 7). Es konnte gezeigt werden, dass IMT die Atemmuskelfunktion (Kraft und Ausdauer) und die körperliche Leistungsfähigkeit verbessern und die Dyspnoe reduzieren kann [5,7,52,53]. Positive Effekte setzen allerdings eine ausreichende Trainingsintensität voraus (siehe oben) [5]. Zumeist kommen threshold load oder resistive load 30 Minuten täglich - oder kürzer - zum Einsatz [5 - 7, $9,10,52]$. Normokapnische Hyperpnoe scheint bei COPD weniger ausgeprägte Verbesserungen erzielen zu können [5]. Ein direkter Vergleich der verschiedenen Trainingsmethoden innerhalb einer Studie wurde allerdings bislang nicht durchgeführt.

Besonders effektiv scheint das IMT bei Patienten mit deutlicher Schwäche der Atemmuskulatur (PImax $<60 \mathrm{cmH}_{2} \mathrm{O}$ ) zu sein [5]. Zusätzliche Effekte eines IMT in Kombination mit körperlichem Training in Bezug auf Dyspnoe und körperliche Leistungsfähigkeit wurden bislang noch nicht eindeutig nachgewiesen $[5,54$, 55].

Auch verbessert IMT mittels threshold load bei COPD die Kontraktionsgeschwindigkeit der inspiratorischen Muskulatur und erhöht den Querschnitt der schnell kontrahierenden (Typ II) Muskelfasern [56,57]. Erste Daten zeigen eine Zunahme der inspiratorischen Kapazität [58] wie auch des Atemzugvolumens [59] während Belastungstests. Dies resultiert in einem effizienteren (langsameren und „tieferen“) Atemmuster. Dieses effizientere Atemmuster [58-60] in Kombination mit verbesserter neuro-mechanischer Kopplung (geringere efferente Aktivierung der Atemmuskulatur) [61] sind Faktoren, die wahrscheinlich zur Verringerung der Dyspnoe bei vergleichbarer Belastung nach IMT beitragen [60]. 

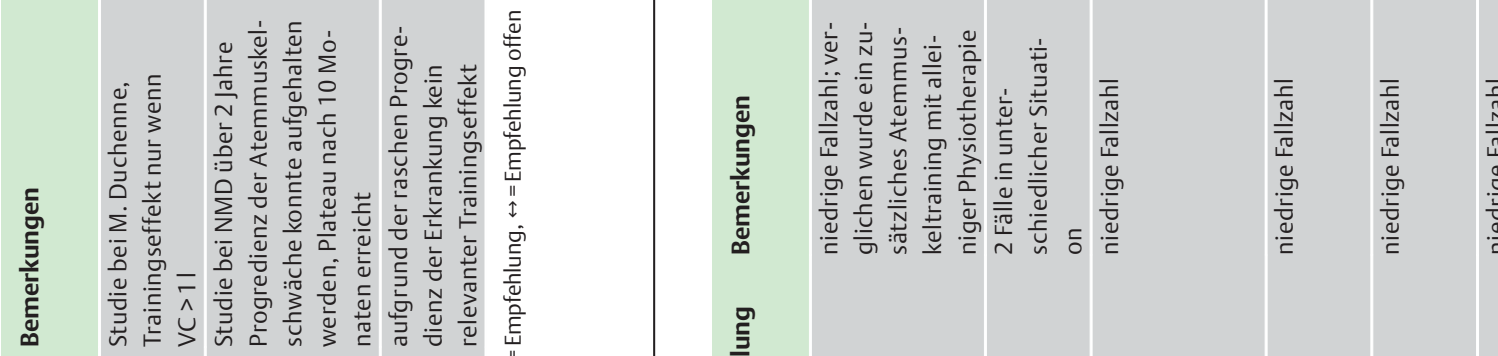

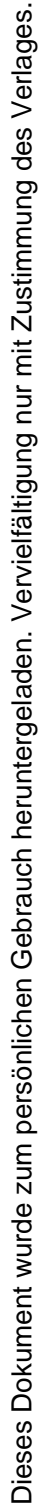
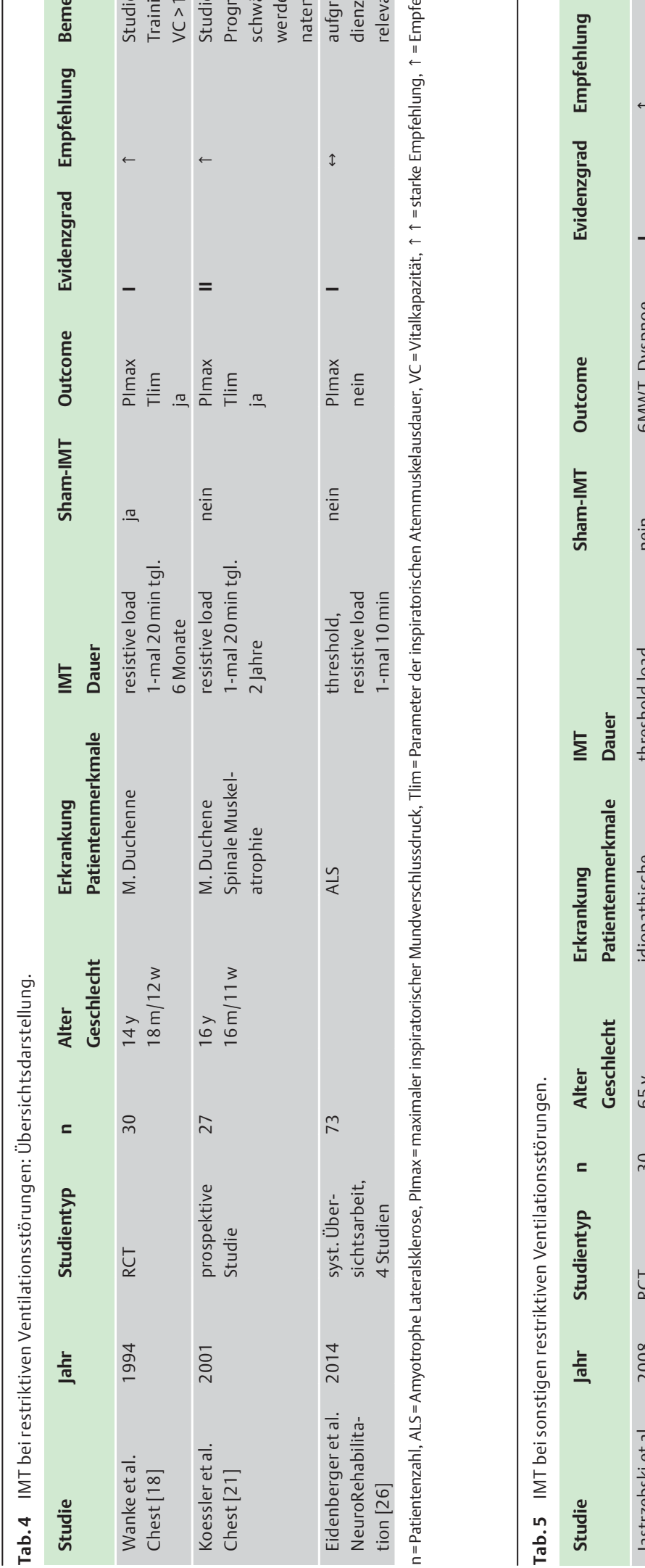

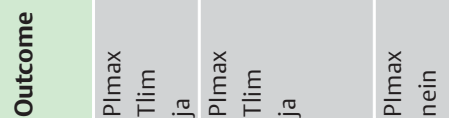
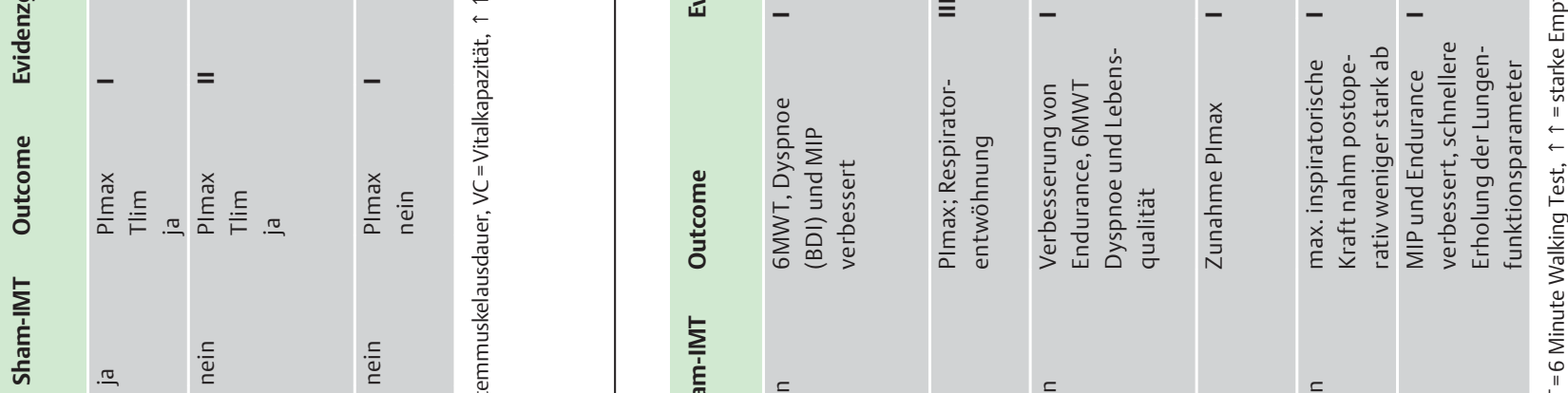

$\stackrel{5}{\underline{5}}$

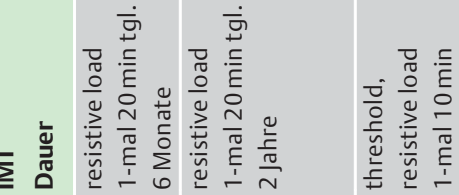<smiles>C1CCC1</smiles><smiles>C[C@@H]1C[C@@H]2CC[C@H]1C2</smiles>

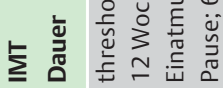

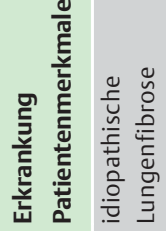

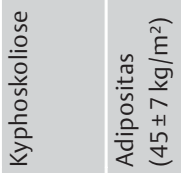
$\therefore \quad$ 듬
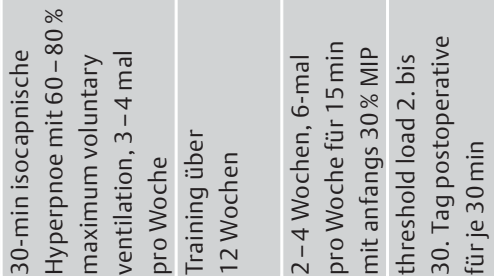

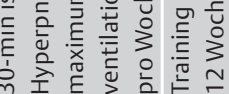

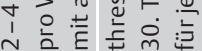
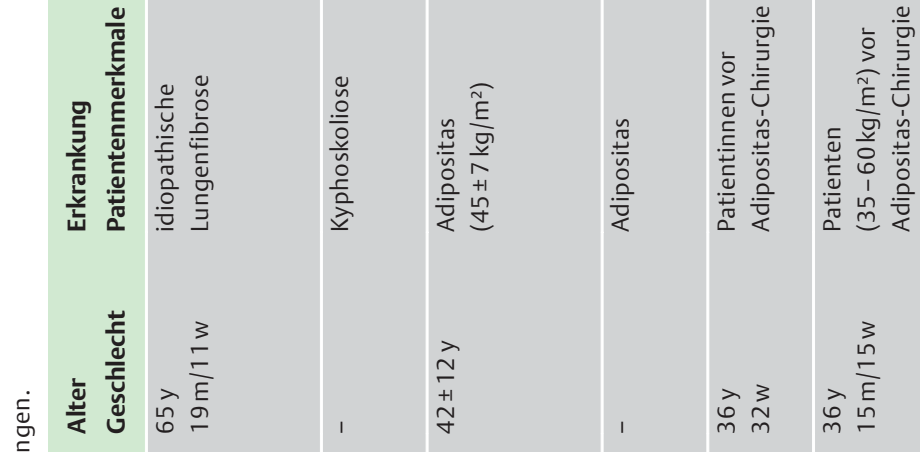

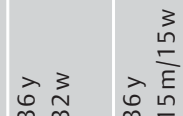

$$
\text { | }
$$

矛

喜<smiles>[SiH3]</smiles>

产

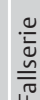
品 


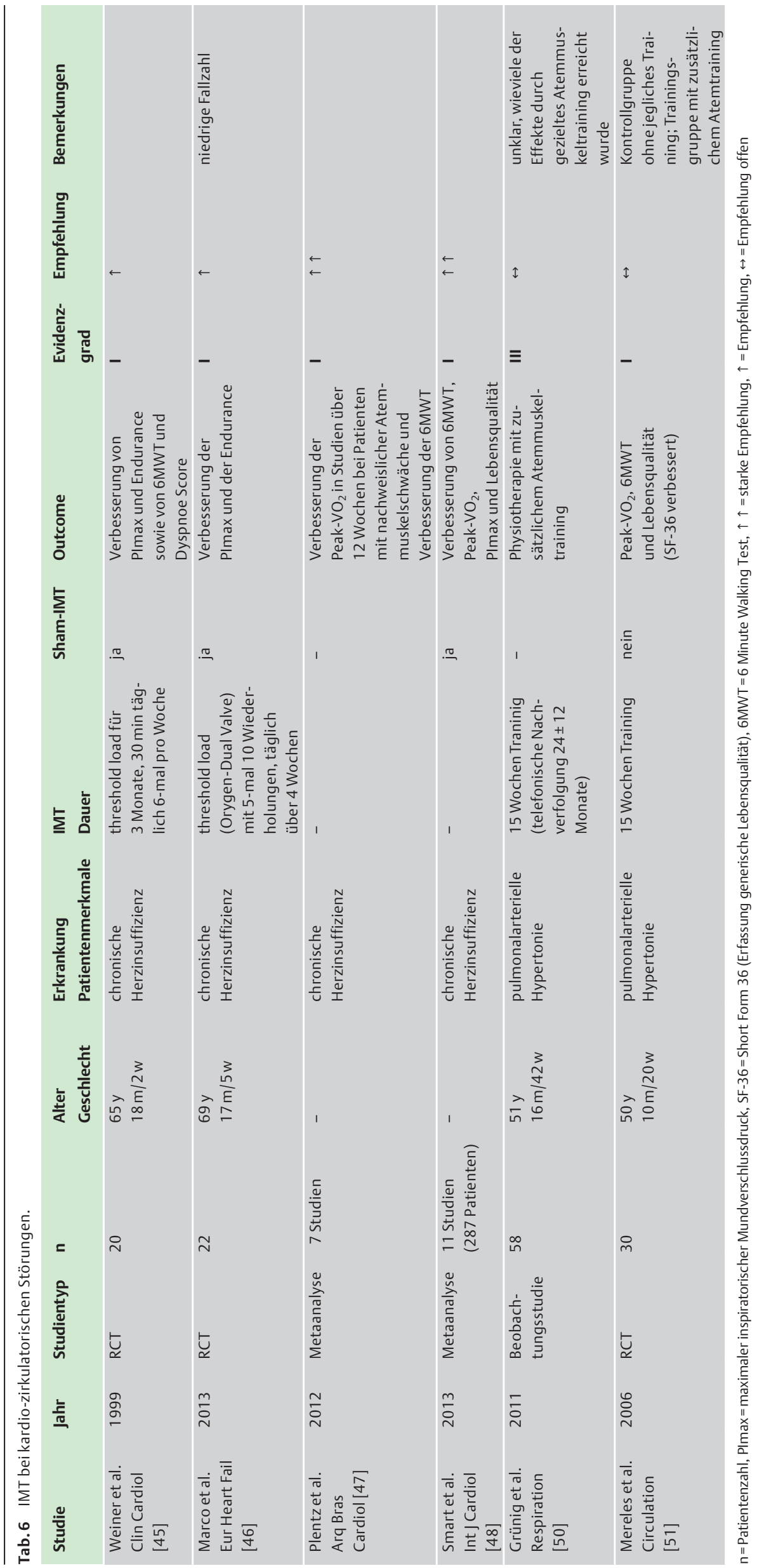




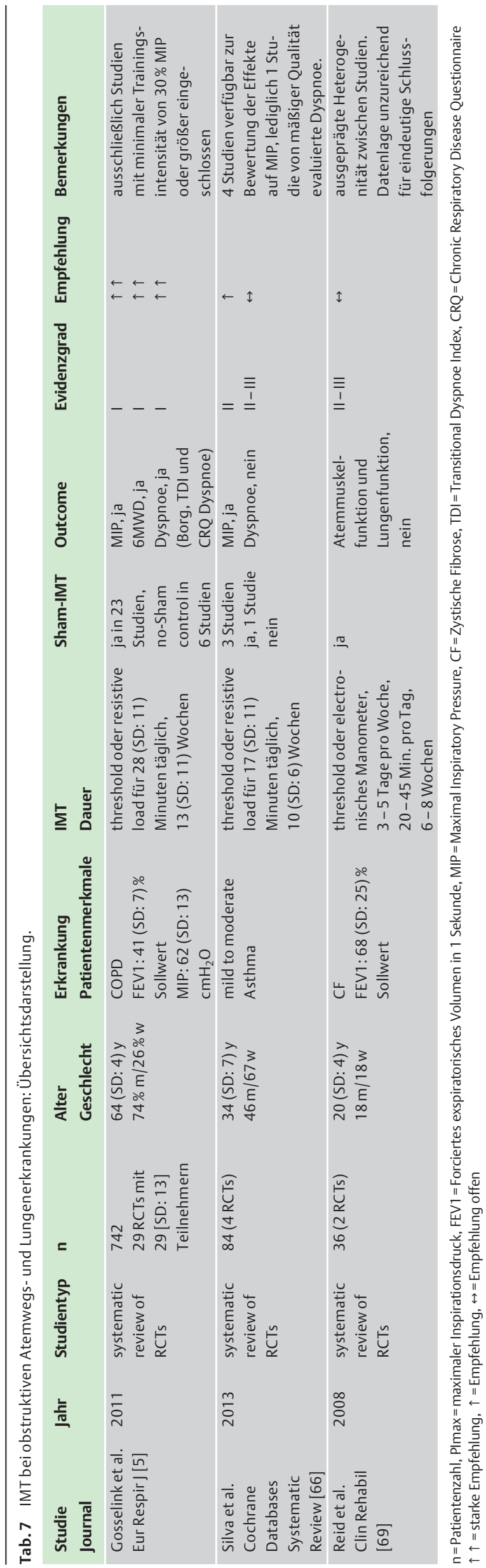

\section{Asthma}

Die Atemmuskelfunktion (Kraft und Ausdauer) scheint bei nicht steroidpflichtigen Asthmatikern im Vergleich zur COPD weniger stark beeinträchtigt zu sein [62-65].

IMT in Form von threshold load oder resistive load verbessert beim Asthma den PImax, ohne jedoch die Dyspnoe zu reduzieren [66] (vgl. @ Tab.7). Aktuell ist die Datenlage jedoch nicht ausreichend, um Add-on-Effekte von IMT, über eine Verbesserung der PImax hinaus, bei Asthma bewerten zu können.

\section{Cystische Fibrose (CF)}

Hier können unzureichende Nährstoffaufnahme und ein persistent kataboler Stoffwechsel aufgrund chronischer Infektionen zur peripheren und respiratorischen Muskelatrophie beitragen $[67,68]$. Als Resultat wird, wie bei Patienten mit COPD, bei vorliegender höherer Belastung der Atemmuskulatur häufig eine Schwächung der Atemmuskulatur beobachtet [67,68].

Trotz der soliden pathophysiologischen Grundlage für IMT ist die Datenlage hier bislang unzureichend $[69,70]$ (vgl. @ Tab.7). Threshold load wurde in Kombination mit körperlichem Training angewendet und zeigte bestenfalls einen Anstieg des PImax, jedoch nicht der Dyspnoe oder Lebensqualität $[71,72]$.

\section{Non-CF Bronchiektasen}

Bei ähnlicher Pathophysiologie wie CF wird ein IMT empfohlen [73]. In zwei randomisierten kontrollierten Studien zeigte ein mehrwöchiges IMT allerdings lediglich eine Verbesserung der Atemmuskelkraft, aber keine statistisch signifikante Verbesserung der 6-Minuten-Gehstrecke gegenüber den Kontrollgruppen $[74,75]$.

\section{Weaning vom Respirator \\ $\nabla$}

Die Notwendigkeit einer (invasiven) Beatmungstherapie führt via komplexer Pathophysiologie zu einer Reduktion der Atemmuskelkraft. Der beatmungsinduzierte Muskelschaden wird als VIDD (ventilator induced diaphragmatic dysfunction) bezeichnet [76]. Mit Zunahme der Weaningzeit steigt auch die Mortalität $[77,78]$. Die Zunahme der atemmuskulären Kraft stellt den besten Prädiktor für den Weaningerfolg dar [79].

Atemmuskeln sind auch bei hyperkapnischen Patienten grundsätzlich trainierbar [80]. Essenziell scheint besonders im Weaning die intermittierende Entlastung via (nicht-) invasiver Beatmung zu sein [81]. Bereits Ende der 1980er Jahre wurden erste Weaningerfolge unter Anwendung eines „IMT“ (nicht-linearer Resistor) beschrieben, welche später mittels threshold load bestätigt wurden [82-84]. Hierbei fehlten jedoch Kontrollgruppen. Eine bahnbrechende Studie, welche eine Kontrollgruppe inkludierte, zeigte bei postoperativen Patienten ebenso beachtliche Weaningerfolge bei Anwendung von threshold load (4-mal tgl. 6-10 Atemzüge) [85]. Kürzlich konnte für internistische Patienten im Weaning durch IMT zwar eine verbesserte Atemmuskelkraft jedoch keine Verbesserung der Extubationsrate gezeigt werden [86]. Kritikpunkte sind hierbei jedoch die möglicherweise zu geringe Last des IMT (40\% PImax) sowie diskussionswürdige Weaning-Strategien [86].

Trotz unzureichender Datenlage zeichnet sich ab, dass IMT im Weaning in entsprechend erfahrenen Behandlungsteams möglich und sicher ist und insbesondere bei Patienten im prolongierten Weaning einen besonderen Benefit aufweisen könnte (vgl. - Tab. 8). 


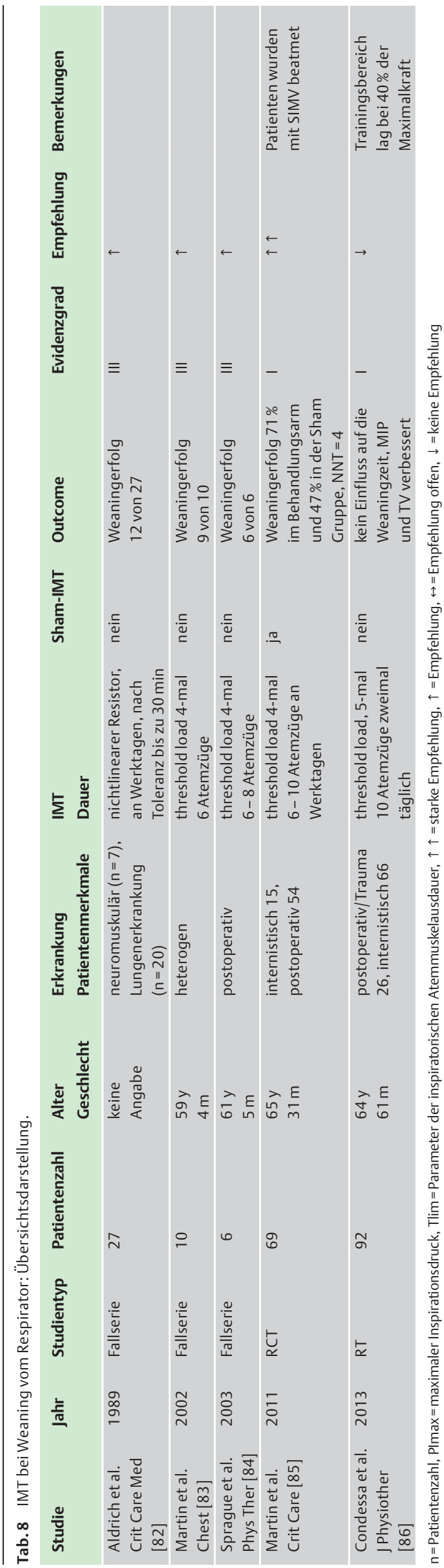

Technische Aspekte und praktische Hinweise

Im Weaning erfolgt IMT fast ausschließlich via künstlichem Atemweg (Tracheostoma). Das IMT-Device wird via Adapter mit der Trachealkanüle (Tubus) verbunden. Der Cuff muss geblockt sein, eine etwaige Sauerstoffzufuhr patientenfern erfolgen (ansonsten Druckverlust und ineffektives IMT). Nur kooperationsfähige Patienten dürfen nach entsprechender Aufklärung einem IMT zugeführt werden. Das IMT muss unter ständiger Überwachung des Patienten erfolgen und ist bei Missempfindungen seitens des Patienten (Dyspnoe, Schmerzen, Angst, etc.) ggf. vorzeitig zu beenden.

\section{Körperliche Aktivität \\ $\nabla$}

Körperliche Belastung erhöht bei Gesunden häufig die Wahrnehmung von Atmungsanstrengung und führt zum Gefühl von Dyspnoe $[6,87]$. Sportliche Tätigkeiten, seien es intensive, kurze Belastungen ( $\geq 85 \%$ der maximalen Sauerstoffaufnahme) oder auch niedriger intensive, längerdauernde Belastungen („Ultramarathon“" u.a.) führen bei rund $70 \%$ der Personen zur Ermüdung der inspiratorischen und/oder exspiratorischen Atemmuskulatur [88 -96]. Eine ermüdete Atemmuskulatur beeinträchtigt umgekehrt eine nachfolgende sportliche Leistung.

Während körperlicher Aktivität und Sport wird die Atmungsmuskulatur häufig „doppelt“ belastet: Neben der Atemaktivität werden die Muskeln einerseits zur Stabilisierung und andererseits für die effektive Bewegung eingesetzt (z. B. Rudern, Schwimmen, aber auch Laufen u.a.) [97]. Die Körperstabilität wird bei ermüdeter Atmungsmuskulatur beeinträchtigt, was die Stolper- $\mid$ Sturzgefahr erhöhen kann [98].

Durch IMT lässt sich beim Gesunden die Ermüdbarkeit der Atmungsmuskulatur reduzieren [99-101]. Mehrheitlich wird durch IMT die Dyspnoe reduziert und die Leistung verbessert (vgl. O Tab.9), was zwei neuere, systematische Reviews mit Meta-Analyse gezeigt haben [6,87]. Entscheidend für den Wirksamkeitsnachweis des IMT hinsichtlich Leistungsverbesserung scheint allerdings die Testart zu sein: Die Leistung in Stufentests zeigt häufig keine Verbesserung, während sie in sogenannten "Time Trials“ und „Constant Load Tests“ mehrheitlich zunimmt $[6,87]$. Auch der Fitnesszustand scheint hier eine Rolle zu spielen, indem weniger Trainierte mehr profitieren [6].

Bezüglich der Wahl der optimalen IMT-Methode zeichnet sich aktuell keine eindeutige Überlegenheit einer Methode ab $[6,87]$. Gegebenenfalls bietet bei Sportarten mit hoher ventilatorischer Anforderung (z.B. Radfahren, Laufen, Langlauf etc.) ein forciertes in- und exspiratorisches Training gegenüber dem rein inspiratorischen Training in Bezug auf die Leistungsverbesserung gewisse Vorteile $[6,87]$. 


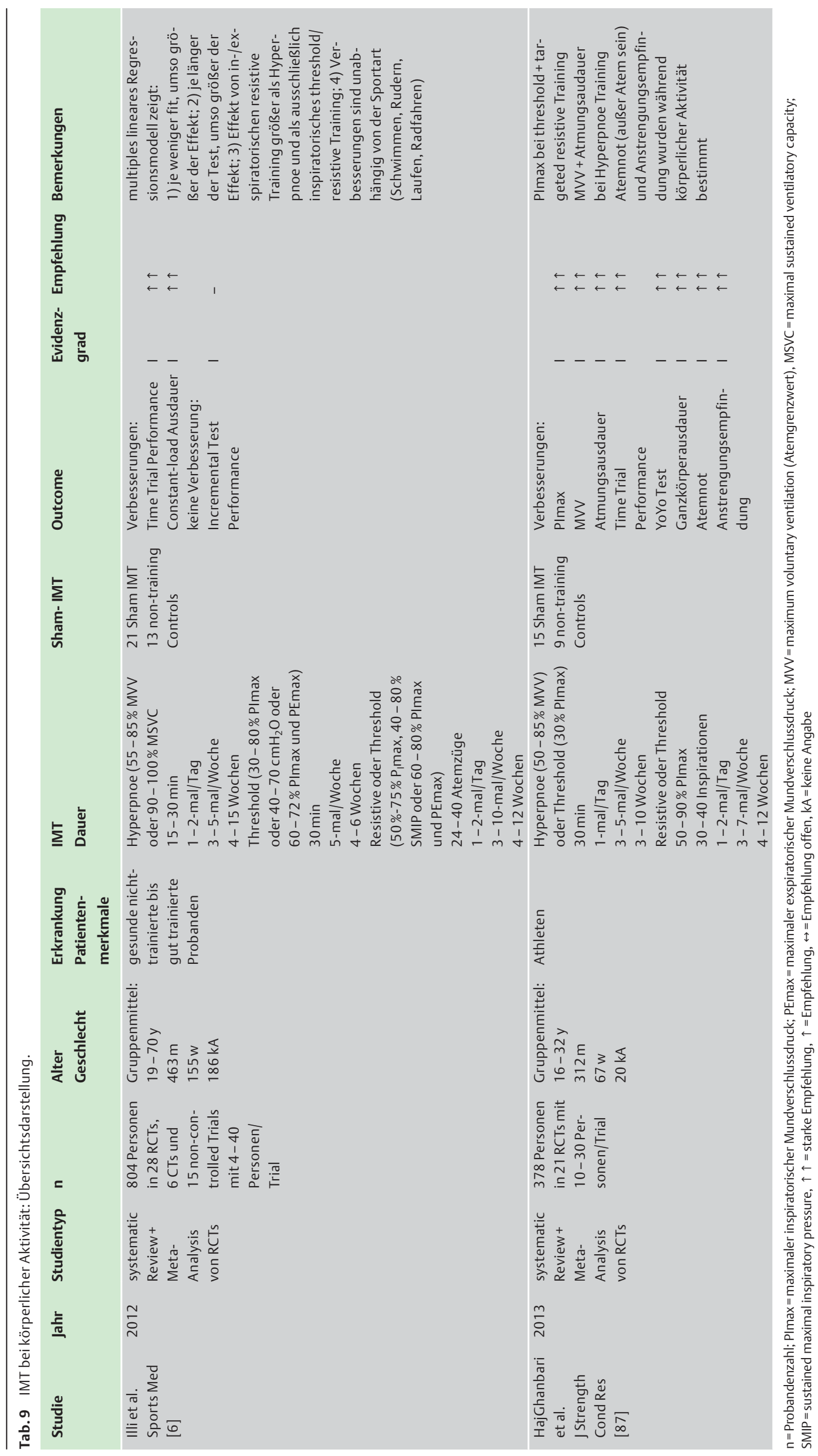


Zusammenfassung

Spezifisches Atemmuskeltraining (IMT) kann anhand der Datenlage aktuell in 3 „methodisch gesicherte“ Verfahren unterteilt werden:

1. Resistive Load

2. Threshold Load

3. Normokapnische Hyperpnoe

Eine eindeutige Überlegenheit eines Verfahrens existiert bis dato nicht; vielmehr müssen die jeweiligen Vor- und Nachteile der Methoden kritisch gegeneinander abgewogen werden. Bezüglich der hier abgehandelten Krankheitsbilder und Indikationen erscheint an vielen Stellen die Datenlage äußerst spärlich, sodass zumeist keine pauschalen Empfehlungen gegeben werden können. Häufig erscheint die Durchführung eines IMT auch aus pathophysiologischen Überlegungen sinnvoll und kann im Einzelfall zur Anwendung kommen.

Stets setzt ein IMT jedoch eine vorherige Diagnostik der Atemmuskelfunktion und eine klare Indikationsstellung voraus. Zum Teil ist es auch unerlässlich, dass IMT lediglich in erfahrenen Behandlungsteams und entsprechender Patientenüberwachung zum Einsatz kommt (z. B. IMT beim Weaning vom Respirator).

Bei gegebener Indikation, korrekter Patientenschulung und guter Compliance erscheint IMT bei vielen Krankheitsbildern eine wertvolle Therapiestrategie zu sein, von der zu wünschen bleibt, dass sie zukünftig häufiger ausgeschöpft werden wird.

\section{Interessenkonflikt}

O. Göhl, D. J. Walker und S. Stieglitz erhielten Vortragshonorare von der Firma Heinen + Löwenstein GmbH \& Co. KG.

H.-J. Kabitz erhielt von den folgenden Firmen Honorare für Vortragstätigkeiten/Reisesponsoring zu wissenschaftlichen Veranstaltungen: Heinen + Löwenstein GmbH\&Co. KG, Bad Ems; VIVISOL Deutschland GmbH, Neufahrn b. Freising; Anamed GmbH\& Co.KG, Bad Ems; Sapio Life GmbH\&Co.KG, Homburg; idiag AG, Fehraltorf (Schweiz).

S. Walterspacher, D. Langer, C. M. Spengler, T. Wanke, M. Petrovic, R.-H. Zwick, R. Glöckl und D. Dellweg geben an, dass kein Interessenkonflikt besteht.

\section{Institute}

${ }^{1}$ Rehaklinik Heidelberg-Königstuhl, Heidelberg

II. Medizinische Klinik, Pneumologie, Kardiologie und Intensivmedizin, Klinikum Konstanz

${ }^{3}$ KU Leuven - University of Leuven, Department of Rehabilitation Sciences, Leuven, Belgien

${ }^{4}$ Exercise Physiology Lab, Institut für Bewegungswissenschaften und Sport, ETH Zürich, und Zentrum für Integrative Humanphysiologie, Universität Zürich, Schweiz

Krankenhaus Hietzing mit Neurologischem Zentrum Rosenhügel und Karl Landsteiner Institut, Abteilung für Atmungs- und Lungenerkrankungen, Wien, Österreich

${ }^{6}$ Univ. Klinik für Innere Medizin, Univ. Klinikum Tulln, Karl Landsteiner Privatuniversität, Ambulante Pneumologische Rehabilitation, Therme Wien Med, Wien, Österreich

7 Medizinische Klinik I - Pneumologie und Kardiologie, Petrus Krankenhaus Wuppertal

Schön Klinik Berchtesgadener Land, Fachzentrum Pneumologie, Schönau am Königssee

${ }^{9}$ Fachkrankenhaus Kloster Grafschaft GmbH, Lehrkrankenhaus der Universität Marburg, Schmallenberg

\section{Literatur}

1 Kabitz HJ, Walterspacher S, Mellies U et al. [Recommendations for respiratory muscle testing]. Pneumologie 2014; 68: 307 - 314

2 Kabitz HJ, Windisch W. Diagnostik der Atemmuskelfunktion: state of the art. Pneumologie 2007; 61: $582-587$

3 Thews G. Lungenatmung. In: Schmidt RF, Thews G Hrsg. Physiologie des Menschen. Berlin, Heidelberg, New York, Tokio: Springer; 1997: $565-591$

4 Leith DE, Bradley $M$. Ventilatory muscle strength and endurance training. J Appl Physiol 1976; 41: 508-516

5 Gosselink R, De Vos J, van den Heuvel SP et al. Impact of inspiratory muscle training in patients with COPD: what is the evidence? Eur Respir J 2011; 37: $416-425$

6 Illi SK, Held U, Frank I et al. Effect of respiratory muscle training on exercise performance in healthy individuals: a systematic review and meta-analysis. Sports Med 2012; 42: 707 - 724

7 Geddes EL, O'Brien K, Reid WD et al. Inspiratory muscle training in adults with chronic obstructive pulmonary disease: an update of a systematic review. Respir Med 2008; 102: 1715-1729

8 Nici L, Donner C, Wouters E et al. American Thoracic Society/European Respiratory Society statement on pulmonary rehabilitation. Am J Respir Crit Care Med 2006; 173: 1390 - 1413

9 Belman MJ, Shadmehr R. Targeted resistive ventilatory muscle training in chronic obstructive pulmonary disease. J Appl Physiol (Bethesda, Md: 1985) 1988; 65: 2726-2735

10 Gosselink R, Wagenaar RC, Decramer $M$. Reliability of a commercially available threshold loading device in healthy subjects and in patients with chronic obstructive pulmonary disease. Thorax 1996; 51: 601 605

11 Caine MP, McConnell AK. Development and evaluation of a pressure threshold inspiratory muscle trainer for use in the context of sports performance. Sports Engineering 2000; 3: 149-159

12 McConnell AK, Romer LM. Dyspnoea in health and obstructive pulmonary disease: the role of respiratory muscle function and training. Sports Med 2004; 34: 117 - 132

13 Scherer TA, Spengler CM, Owassapian D et al. Respiratory muscle endurance training in chronic obstructive pulmonary disease: impact on exercise capacity, dyspnea, and quality of life. Am J Respir Crit Care Med 2000; 162: 1709-1714

14 Slavka G. Das motorische Nervensystem. Wien (Grafik unpubliziert) 2015

15 Walton J. Clinical Examination, Differential Diagnosis and Classification. In: Walton J, Karpati G, Hilton DJ eds. Disorders of voluntary muscle. 6th ed. Edinburgh: Churchill Livingstone; 1994: 547

16 Mah JK, Korngut L, Dykeman J et al. A systematic review and meta-analysis on the epidemiology of Duchenne and Becker muscular dystrophy. Neuromuscul Disord 2014; 24: 482 - 491

17 DiMarco AF, Kelling JS, DiMarco MS et al. The effects of inspiratory resistive training on respiratory muscle function in patients with muscular dystrophy. Muscle Nerve 1985; 8: 284-290

18 Wanke T, Toifl K, Merkle $M$ et al. Inspiratory muscle training in patients with Duchenne muscular dystrophy. Chest 1994; 105: 475-482

19 Winkler G, Zifko U, Nader A et al. Dose-dependent effects of inspiratory muscle training in neuromuscular disorders. Muscle Nerve 2000; 23: $1257-1260$

20 Topin N, Matecki S, Le Bris S et al. Dose-dependent effect of individualized respiratory muscle training in children with Duchenne muscular dystrophy. Neuromuscul Disord 2002; 12: 576-583

21 Koessler W, Wanke T, Winkler G et al. 2 Years' experience with inspiratory muscle training in patients with neuromuscular disorders. Chest 2001; 120: $765-769$

22 Smith PE, Coakley JH, Edwards RH. Respiratory muscle training in Duchenne muscular dystrophy. Muscle Nerve 1988; 11: 784-785

23 Aslan GK, Gurses HN, Issever $H$ et al. Effects of respiratory muscle training on pulmonary functions in patients with slowly progressive neuromuscular disease: a randomized controlled trial. Clin Rehabil 2013; 28: $573-581$

24 Gozal D, Thiriet P. Respiratory muscle training in neuromuscular disease: long-term effects on strength and load perception. Med Sci Sports Exerc 1999; 31: $1522-1527$

25 Pinto $S$, de Carvalho $M$. Can inspiratory muscle training increase survival in early-affected amyotrophic lateral sclerosis patients? Amyotroph Lateral Scler Frontotemporal Degener 2013; 14: 124-126 
26 Eidenberger $M$, Nowotny $S$. Inspiratory muscle training in patients with amyotrophic lateral sclerosis: A systematic review. NeuroRehabilitation 2014; 35: 349 - 361

27 Petrovic M, Lahrmann H, Pohl W et al. Idiopathic diaphragmatic paralysis - satisfactory improvement of inspiratory muscle function by inspiratory muscle training. Respir Physiol Neurobiol 2009; 165: 266-267

28 Mueller G, Perret C, Spengler CM. Optimal intensity for respiratory muscle endurance training in patients with spinal cord injury. J Rehabil Med 2006; 38: 381 - 386

29 Van Houtte S, Vanlandewijck Y, Kiekens C et al. Patients with acute spinal cord injury benefit from normocapnic hyperpnoea training. J Rehabil Med Medicine 2008; 40: 119-125

30 Berlowitz DJ, Tamplin J. Respiratory muscle training for cervical spinal cord injury. Cochrane Database Syst Rev 2013; 7: Cd008507

31 Watanabe F, Taniguchi H, Sakamoto K et al. Quadriceps weakness contributes to exercise capacity in nonspecific interstitial pneumonia. Respir Med 2013; 107: 622-628

32 Jastrzebski D, Kozielski J, Zebrowska A. [Pulmonary rehabilitation in patients with idiopathic pulmonary fibrosis with inspiratory muscle training]. Pneumonologia i alergologia polska 2008; 76: $131-141$

33 Newman LS, Rose CS, Maier LA. Sarcoidosis. N Engl J Med 1997; 336: $1224-1234$

34 Kabitz HJ, Lang F, Walterspacher S et al. Impact of impaired inspiratory muscle strength on dyspnea and walking capacity in sarcoidosis. Chest 2006; 130: 1496 - 1502

35 Murray J, Nadel J. Textbook of respiratory medicine. $3 \mathrm{rd}$ ed. Philadelphia: Saunders; 2000

36 Hornstein S, Inman S, Ledsome JR. Ventilatory muscle training in kyphoscoliosis. Spine 1987; 12: 859-863

37 Kelly TM, Jensen RL, Elliott CG et al. Maximum respiratory pressures in morbidly obese subjects. Respirations 1988; 54: 73 -77

38 Chlif M, Keochkerian D, Choquet $D$ et al. Effects of obesity on breathing pattern, ventilatory neural drive and mechanics. Respir Physiol Neurobiol 2009; 168: 198-202

39 Villiot-Danger JC, Villiot-Danger E, Borel JC et al. Respiratory muscle endurance training in obese patients. Int J Obes (2005) 2011; 35: 692 699

40 Tenorio $\mathrm{LH}$, Santos AC, Camara Neto JB et al. The influence of inspiratory muscle training on diaphragmatic mobility, pulmonary function and maximum respiratory pressures in morbidly obese individuals: a pilot study. Disabil Rehabil 2013; 35: 1915-1920

41 Barbalho-Moulim MC, Miguel GP, Forti EM et al. Effects of preoperative inspiratory muscle training in obese women undergoing open bariatric surgery: respiratory muscle strength, lung volumes, and diaphragmatic excursion. Clinics (Sao Paulo, Brazil) 2011; 66: 1721 - 1727

42 Casali CC, Pereira AP, Martinez JA et al. Effects of inspiratory muscle training on muscular and pulmonary function after bariatric surgery in obese patients. Obesity Surgery 2011; 21: 1389-1394

43 McParland C, Krishnan B, Wang Y et al. Inspiratory muscle weakness and dyspnea in chronic heart failure. Am Rev Respir Dis 1992; 146: 467-472

44 Nishimura $Y$, Maeda $H$, Tanaka K et al. Respiratory muscle strength and hemodynamics in chronic heart failure. Chest 1994; 105: 355 - 359

45 Weiner P, Waizman J, Magadle R et al. The effect of specific inspiratory muscle training on the sensation of dyspnea and exercise tolerance in patients with congestive heart failure. Clin Cardiol 1999; 22: 727 - 732

46 Marco E, Ramirez-Sarmiento AL, Coloma A et al. High-intensity vs. sham inspiratory muscle training in patients with chronic heart failure: a prospective randomized trial. Eur J Heart Fail 2013; 15: 892 - 901

47 Plentz RD, Sbruzzi G, Ribeiro RA et al. Inspiratory muscle training in patients with heart failure: meta-analysis of randomized trials. Arq Bras Cardiol 2012; 99: 762-771

48 Smart NA, Giallauria F, Dieberg G. Efficacy of inspiratory muscle training in chronic heart failure patients: a systematic review and metaanalysis. Int J Cardiol 2013; 167: 1502 - 1507

49 Kabitz HJ, Schwoerer A, Bremer HC et al. Impairment of respiratory muscle function in pulmonary hypertension. Clin Sci (Lond) 2008; 114: 165 - 171

50 Grunig E, Ehlken N, Ghofrani A et al. Effect of exercise and respiratory training on clinical progression and survival in patients with severe chronic pulmonary hypertension. Respiration 2011; 81: 394-401

51 Mereles D, Ehlken N, Kreuscher $S$ et al. Exercise and respiratory training improve exercise capacity and quality of life in patients with severe chronic pulmonary hypertension. Circulation 2006; 114: 1482 -1489
52 Shoemaker MJ, Donker S, Lapoe A. Inspiratory muscle training in patients with chronic obstructive pulmonary disease: the state of the evidence. Cardiopulm Phys Ther J 2009; 20: 5 - 15

53 Geddes EL, Reid WD, Crowe J et al. Inspiratory muscle training in adults with chronic obstructive pulmonary disease: a systematic review. Respir Med 2005; 99: 1440-1458

54 Ambrosino $N$. The case for inspiratory muscle training in COPD. For. Eur Respir J 2011; 37: 233-235

55 Polkey MI, Moxham J, Green M. The case against inspiratory muscle training in COPD. Against. Eur Respir J 2011; 37: 236-237

56 Ramirez-Sarmiento A, Orozco-Levi M, Guell R et al. Inspiratory muscle training in patients with chronic obstructive pulmonary disease: structural adaptation and physiologic outcomes. Am J Respir Crit Care Med 2002; 166: 1491 - 1497

57 Villafranca C, Borzone G, Leiva A et al. Effect of inspiratory muscle training with an intermediate load on inspiratory power output in COPD. Eur Respir J 1998; 11: 28 - 33

58 Petrovic $M$, Reiter $M$, Zipko $H$ et al. Effects of inspiratory muscle training on dynamic hyperinflation in patients with COPD. Int J Chron Obstruct Pulmon Dis 2012; 7: $797-805$

59 Charususin $N$, Langer D, Gosselink R. Does additional inspiratory muscle training alter breathing pattern during whole body exercise in COPD patients? Eur Respir J 2014; 44: 3345

60 Macklem PT. Therapeutic implications of the pathophysiology of COPD Eur Respir J 2010; 35: 676-680

61 Langer D, Ciavaglia C, Preston $M$ et al. Inspiratory Muscle Training Reduces Respiratory Neural Drive In Patients With COPD. Eur Respir J 2014; 44: 1912

62 Perez T, Becquart LA, Stach B et al. Inspiratory muscle strength and endurance in steroid-dependent asthma. Am J Respir Crit Care Med 1996; 153: $610-615$

63 Stell IM, Polkey MI, Rees PJ et al. Inspiratory muscle strength in acute asthma. Chest 2001; 120: 757-764

64 Lavietes MH, Grocela JA, Maniatis T et al. Inspiratory muscle strength in asthma. Chest 1988; 93: 1043-1048

65 McKenzie DK, Gandevia SC. Strength and endurance of inspiratory, expiratory, and limb muscles in asthma. Am Rev Respir Dis 1986; 134 999- 1004

66 Silva IS, Fregonezi GA, Dias FA et al. Inspiratory muscle training for asthma. Cochrane Database Syst Rev 2013; 9: CD003792

67 Pinet C, Cassart M, Scillia $P$ et al. Function and bulk of respiratory and limb muscles in patients with cystic fibrosis. Am J Respir Crit Care Med 2003; 168: $989-994$

68 Ionescu $A A$, Chatham $K$, Davies $C A$ et al. Inspiratory muscle function and body composition in cystic fibrosis. Am J Respir Crit Care Med 1998; 158: 1271 - 1276

69 Reid WD, Geddes EL, O'Brien K et al. Effects of inspiratory muscle training in cystic fibrosis: a systematic review. Clin Rehabil 2008; 22: $1003-1013$

70 Santana-Sosa E, Gonzalez-Saiz L, Groeneveld IF et al. Benefits of combining inspiratory muscle with 'whole muscle' training in children with cystic fibrosis: a randomised controlled trial. Br J Sports Med 2014; 48: $1513-1517$

71 Enright $S$, Chatham $K$, Ionescu $A A$ et al. Inspiratory muscle training improves lung function and exercise capacity in adults with cystic fibrosis. Chest 2004; 126 : $405-411$

72 de Jong $W$, van Aalderen WM, Kraan J et al. Inspiratory muscle training in patients with cystic fibrosis. Respir Med 2001; 95: 31 - 36

73 Rochester CL, Fairburn C, Crouch $R H$. Pulmonary rehabilitation for respiratory disorders other than chronic obstructive pulmonary disease. Clin Chest Med 2014; 35: 369-389

74 Newall C, Stockley RA, Hill SL. Exercise training and inspiratory muscle training in patients with bronchiectasis. Thorax 2005; 60: 943 -948

75 Liaw MY, Wang YH, Tsai YC et al. Inspiratory muscle training in bronchiectasis patients: a prospective randomized controlled study. Clin Rehabil 2011; 25: 524-536

76 Levine S, Nguyen T, Taylor $N$ et al. Rapid Disuse Atrophy of Diaphragm Fibers in Mechanically Ventilated Humans. N Engl J Med 2008; 358: $1327-1335$

77 Esteban A, Anzueto A, Frutos F et al. Characteristics and outcomes in adult patients receiving mechanical ventilation: a 28-day international study. JAMA 2002; 287: 345 - 355

78 Funk GC, Anders S, Breyer MK et al. Incidence and outcome of weaning from mechanical ventilation according to new categories. Eur Respir J 2010; 35 : $88-94$ 
79 Carlucci A, Ceriana P, Prinianakis $G$ et al. Determinants of weaning success in patients with prolonged mechanical ventilation. Crit Care 2009; 13: R97

80 Budweiser S, Moertl M, Jorres RA et al. Respiratory muscle training in restrictive thoracic disease: a randomized controlled trial. Arch Phys Med Rehabil 2006; 87: 1559-1565

81 Schonhofer B, Geiseler J, Dellweg D et al. [Prolonged weaning: S2kguideline published by the German Respiratory Society]. Pneumologie 2014; 68: 19-75

82 Aldrich TK, Karpel JP, Uhrlass RM et al. Weaning from mechanical ventilation: adjunctive use of inspiratory muscle resistive training. Crit Care Med 1989; 17: 143-147

83 Martin AD, Davenport PD, Franceschi $A C$ et al. Use of inspiratory muscle strength training to facilitate ventilator weaning: a series of 10 consecutive patients. Chest 2002; 122: 192 - 196

84 Sprague SS, Hopkins PD. Use of inspiratory strength training to wean six patients who were ventilator-dependent. Phys Ther 2003; 83: $171-181$

85 Martin AD, Smith BK, Davenport PD et al. Inspiratory muscle strength training improves weaning outcome in failure to wean patients: a randomized trial. Crit Care (London, England) 2011; 15: R84

86 Condessa RL, Brauner JS, Saul AL et al. Inspiratory muscle training did not accelerate weaning from mechanical ventilation but did improve tidal volume and maximal respiratory pressures: a randomised trial. J Physiother 2013; 59: 101 - 107

87 HajGhanbari B, Yamabayashi C, Buna TR et al. Effects of respiratory muscle training on performance in athletes: a systematic review with meta-analyses. J Strength Cond Res 2013; 27: 1643 -1663

88 Mador MJ, Magalang UJ, Rodis A et al. Diaphragmatic fatigue after exercise in healthy human subjects. Am Rev Respir Dis 1993; 148: 1571 1575

89 Johnson BD, Babcock MA, Suman OE et al. Exercise-induced diaphragmatic fatigue in healthy humans. J Physiol 1993; 460: 385-405
90 Verges $S$, Schulz $C$, Perret $C$ et al. Impaired abdominal muscle contractility after high-intensity exhaustive exercise assessed by magnetic stimulation. Muscle Nerve 2006; 34: 423-430

91 Taylor BJ, How SC, Romer LM. Exercise-induced abdominal muscle fatigue in healthy humans. J Appl Physiol 2006; 100: 1554-1562

92 Romer LM, Polkey MI. Exercise-induced respiratory muscle fatigue: implications for performance. J Appl Physiol 2008; 104: 879-888

93 Wüthrich TU, Marty J, Kerherve $H$ et al. Aspects of respiratory muscle fatigue in a mountain ultramarathon race. Med Sci Sports Exerc 2015; 47: $519-527$

94 Choukroun ML, Kays C, Gioux M et al. Respiratory muscle function in trained and untrained adolescents during short-term high intensity exercise. Eur J Appl Physiol Occup Physiol 1993; 67: 14-19

95 Coast JR, Clifford PS, Henrich TW et al. Maximal inspiratory pressure following maximal exercise in trained and untrained subjects. Med Sci Sports Exerc 1990; 22: $811-815$

96 Babcock MA, Pegelow DF, Johnson BD et al. Aerobic fitness effects on exercise-induced low-frequency diaphragm fatigue. J Appl Physiol 1996; 81: $2156-2164$

97 Gandevia SC, Butler JE, Hodges PW et al. Balancing acts: respiratory sensations, motor control and human posture. Clin Exp Pharmacol Physiol 2002; 29: $118-121$

98 Janssens L, Brumagne S, Polspoel K et al. The effect of inspiratory muscles fatigue on postural control in people with and without recurrent low back pain. Spine 2010; 35: 1088 - 1094

99 Verges $S$, Lenherr $O$, Haner $A C$ et al. Increased fatigue resistance of respiratory muscles during exercise after respiratory muscle endurance training. Am J Physiol Regul Integr Comp Physiol 2007; 292: R12461253

100 Volianitis S, McConnell AK, Koutedakis Yet al. Inspiratory muscle training improves rowing performance. Med Sci Sports Exerc 2001; 33: $803-809$

101 Romer LM, McConnell AK, Jones DA. Inspiratory muscle fatigue in trained cyclists: effects of inspiratory muscle training. Med Sci Sports Exerc 2002; 34: $785-792$ 